\title{
The experiences and perceptions of family planning of female Chinese asylum seekers living in the UK
}

\author{
Alice Verran, ${ }^{1}$ Sarah Evans, ${ }^{2}$ Daniel J Lin, ${ }^{1}$ Frances Griffiths ${ }^{3}$
}

\begin{abstract}
- Additional material is published online only. To view please visit the journal online (http://dx.doi.org/10.1136/fprhc2013-100764)

${ }^{1}$ Medical Student, Warwick Medical School, University of Warwick, Coventry, UK

${ }^{2}$ Research Fellow, Warwick Medical School, University of Warwick, Coventry, UK ${ }^{3}$ Professor of Medicine in Society, Warwick Medical School, University of Warwick, Coventry, UK
\end{abstract}

\section{Correspondence to}

Ms Alice Verran, Warwick Medical School, University of Warwick, Coventry CV4 7AL, UK; a.verran@warwick.ac.uk

Received 18 August 2013 Revised 13 February 2014 Accepted 14 March 2014 Published Online First

17 April 2014

\begin{abstract}
Introduction Chinese family planning policy is unique. There is limited sex education and the state is highly influential. This has resulted in extremely wide coverage of contraception with long-acting methods being favoured. The Chinese constitute a large proportion of asylum applicants to the UK. This study examines how their experiences and decisions about family planning in the UK are shaped by their cultural background.
\end{abstract}

Methods Data were drawn from 10 semistructured qualitative interviews with female Chinese asylum seekers recruited through a family planning clinic in the UK.

Results The increased autonomy provided by the UK system was appreciated by the participants. Choice of contraceptive method was influenced by traditional cultural beliefs and values, and the effect of hormonal contraception on menstruation was particularly concerning. Women arrived from China with little knowledge of contraception. Friends from a similar background were the most trusted source of advice. When transitioning from China to the UK unwanted pregnancies had occurred amongst unmarried women who had missed out on sex education while living in China.

Conclusions Chinese societal and cultural practices continue to influence family planning decisions made within the UK. Culturally competent health strategies are needed to ensure Chinese immigrant women fully benefit from family planning within the UK.

\section{INTRODUCTION}

The Chinese are consistently amongst the UK's top applicant nationalities for asylum ${ }^{1}$ but family planning policy in China and the UK differ vastly. Whereas in the UK it is largely up to the individual to decide when to have children, China has a policy in which the state dictates if,
Key message points

- The Chinese family planning system provides limited sex education and little autonomy to choose what contraception to use or when to have children.

- Cultural beliefs relating to personal health, balance and maintaining natural menstruation may mean that hormonal methods commonly used in the UK are avoided.

- This sociocultural background creates specific family planning needs and tailored health strategies should be developed that ensure early access to services.

when, and how many children a couple may have. ${ }^{2}$ Idiosyncratic beliefs about health and wellbeing within Chinese society are important influences on health-related decision making, ${ }^{3}{ }^{4}$ and may also be expected to influence family planning decisions. It is unknown whether Chinese women's decisions about family planning continue to be influenced by the knowledge and principles acquired in China or whether they become shaped by the norms representative of UK culture and policy. Therefore, although UK policy recognises that good practice guidelines for asylum seeker health should be developed and promoted, ${ }^{5}$ the distinctive sociocultural background of Chinese asylum seekers demands particular consideration regarding how best to support this group of women with family planning services.

Previous studies have demonstrated that migrant women are often disadvantaged in their sexual and reproductive health care. ${ }^{67}$ Language difficulties have 
been identified as a common barrier to appropriate access ${ }^{8} 9$ together with negative attitudes towards certain forms of contraception. ${ }^{10}{ }^{11}$ It is recognised that different cultural backgrounds present distinctive issues due to knowledge and attitudes towards contraception. ${ }^{12}$ An understanding of each group's perceptions is important to appropriately tailor health care services to specific cultural needs. Little is known about the perceptions of Chinese asylum seekers on family planning once in the UK.

Chinese immigrant women are likely to retain values and practices from Chinese culture that may affect their perceptions of family planning. Chinese family planning policy is unusual for its 'one child policy', which has high adherence and, despite recent speculation about its future, remains deeply engrained in Chinese culture and politics. ${ }^{13}$ Most couples are limited to one child although some exceptions do exist. Birth spacing (permits are usually granted after 5 years) and long-acting reversible methods of contraception are fundamental to the success of the policy.

In China, the intrauterine contraceptive device (IUD) is the most commonly used method ${ }^{14}$ and oral hormonal contraceptives have a low acceptability. ${ }^{15}$ In addition, knowledge about sexual and reproductive health is low amongst young unmarried Chinese adults. ${ }^{16-20}$ This means that young Chinese immigrants will typically have a relatively limited understanding of contraception.

Although it is recognised that culturally sensitive services should be developed, once Chinese women move to the UK their health beliefs begin to incorporate Western culture. ${ }^{21-23}$ This study aims to explore their knowledge and understanding of family planning once living in the UK. It was developed to follow up a preliminary study that suggested Chinese asylum seekers experienced problems accessing family planning relating to their cultural and social background.

\section{METHODS}

\section{Research design}

A qualitative study was conducted at a specialist baby clinic for asylum seekers and refugees in the West Midlands, UK. Purposive sampling ${ }^{24}$ and recruitment was carried out by one of the clinic's regular Mandarin interpreters. The inclusion criteria were: being originally from mainland China, Mandarin speaking and with experience of childbearing. Further participants were recruited on the recommendation of interviewees in order to reach women not attending the clinic or at different stages of parenthood. Ten women of childbearing age were recruited. All had given birth in the UK. Three had also given birth in China.

As the participants' level of English was limited yet the research team was English-speaking, Williamson et al.'s ${ }^{25}$ method of interpreter-facilitated crosslanguage interviews was used. The semi-structured interviews were conducted in Mandarin by the interpreter and audio-recorded, and explored attitudes, beliefs, experiences and behaviours related to contraception and family planning.

Brief demographic data were also gathered. Prior training of the interpreter ensured issues were reflectively discussed, particularly any cultural assumptions. Using an interpreter of the same cultural background who had previously met the participants was beneficial for establishing credibility and trust in the research but nevertheless it was recognised that the interviewer's status could influence the participants' openness. $^{26}$

For the translation process, a Mandarin-speaking researcher translated the audio-recordings 'verbatim' into English. The lead researcher engaged the translator in interactive discussion throughout this process to prioritise meaning over literal translation thereby increasing the data's trustworthiness. ${ }^{27}$ Any difficulties with interpretation were discussed and clarified, as were any cultural issues implicit between interviewer and interviewee.

The study was approved by the West Midlands Research Ethics Committee and undertaken in accordance with their guidelines. Informed consent was taken by the interviewer and all participants were given written information in Mandarin and an opportunity to ask questions about the study.

\section{Analysis}

Thematic analysis followed an iterative process of coding and reviewing themes. ${ }^{28}$ We adopted an interpretist approach that prioritised understanding of meaning and the influence of social rules and the host culture. ${ }^{29}$ For these cross-cultural interviews, we conceptualised translation as being more than simply exchanging words between languages. We used those interpreting to bridge the meanings and implicit understandings of those involved at each stage of data production. ${ }^{30}$ Our analysis involved reflection on the meanings attached to participants' accounts of their experiences and perceptions of family planning ${ }^{31}$ and their interaction with their context, in particular with established social practices and community assumptions. $^{32}$

The demographic data were tabulated into categories to provide an overview of the characteristics of the interviewees, and were considered alongside the interview data. In particular, we differentiated between those women who had used family planning services in China and those who had not.

The data were initially coded into 'macro-themes' with two categories forming the top-level coding: 'knowledge of contraception' and 'aspects important in making family planning decisions'. A more detailed coding process was then undertaken, which focused on links between themes and demographics. Through comparison of the data we considered the influences 
on the participants' perceptions about family planning, in particular the influence of cultural context and institutional pressures.

This article includes a small selection of relevant quotations; additional quotes are provided in online Supplementary Table S1.

\section{RESULTS}

\section{Sociodemographic findings}

The participants' mean age was 34.2 (youngest 26, oldest 41) years, and average length of time in the UK was 8.4 years. Their mean age upon arrival in the UK was 25.8 years, with the youngest arriving aged 18 years. All participants had spent time in China whilst of legal childbearing age. All had used contraception whilst in the UK and had two or more children (mean age 6.2 years, range 4 months-24 years) with at least one of them born in the UK. Three participants had given birth in China and four had used contraception while living there.

\section{Family planning knowledge}

Level of knowledge on arrival in the UK

There were important differences between the knowledge of family planning acquired in China and that acquired in the UK. Women who were childless when they came from China appeared to have a very limited knowledge because they had received little sex education in China. Only one participant had used contraception prior to becoming pregnant and she had worked in the health care field. Some participants described how this limited knowledge had led to unplanned pregnancies. [NB. P denotes participant.]

"In China, my parents were strict and I was sheltered and unknowledgeable regarding sexual matters. After coming to the UK and having a sexual relationship with my boyfriend, my understanding ... about intercourse was very limited, so immediately after intercourse, I would go for a pee and thought that would prevent pregnancy." [P8]

Women who had given birth in China described how further pregnancies were prevented through the mandatory use of long-term contraception. They had little choice of contraceptive method and their knowledge of contraception was generally restricted to the IUD. Participants indicated that this lack of access to alternative methods meant that they did not have experience of processing information about different options in order to make decisions themselves.

Transition from Chinese to UK family planning

Once in the UK, family planning information was acquired from a variety of sources. General practice was the most common but hospital-based postnatal and family planning services were also sources. Postnatal advice services were the first point of contact with family planning services for many of the younger women, most of whom had not used contraception prior to pregnancy. The advice of friends from the same cultural background was also highly valued, trusted and frequently sought.

The gap in family planning care that occurred with emigrating had caused problems for some participants. Two of the participants had experienced problems with IUDs fitted in China that then required removal in the UK. For one woman this was delayed because she was not registered with a general practitioner (GP). Misunderstandings about the UK system were described. One participant who was dissatisfied with the implant had not realised that she could see her GP to discuss alternatives and get it removed.

Several participants described difficulties in fully comprehending information provided by health professionals, which limited its use in their decision making.

\begin{abstract}
"The doctor explains to us about issues with contraception failing and we don't entirely understand so it makes us worry ... I still would rather trust my friends' advice on the best method of contraception. If none of my friends can give me an ideal answer, it worries me ... I have received leaflets from doctors but they do not describe adverse reactions. Also, the longterm effects have not been explained to me so I get worried.” [P3]
\end{abstract}

Although some issues were related to language difficulties, they were compounded by cultural misunderstandings. One participant explained that she had used "the removable type" of IUD, but further exploration revealed she had accidentally pulled it out. Concern about side effects was common, particularly relating to hormonal methods. For the oral contraceptive pill, side effects mentioned included reduced energy, hip pain and heart problems, while users of the implant reported fatigue and arm weakness. Many worried about gaining weight when taking hormonal contraceptives. One participant believed the weight gain was due to not menstruating. These concerns led to non-compliance and irregular usage of the oral contraceptive pill, and dissatisfaction with and subsequent removal of the implant.

Some women expected extensive information to inform their decision making and were disappointed with what was offered. This reduced their confidence in the information provided by UK health professionals.

\footnotetext{
"For people like us, who have a language barrier and are attending a GP to get contraception fitted, the most that I am able to understand is probably the effectiveness of the contraceptive and how safe it is, but I would like to know more about the side effects of the implant and the implications of its stopping menstruation.... I'd like to be provided, not only with information on its efficacy, but also its side effects and the most recent research findings on the contraception." [P9]
} 
In summary, information about the health effects of each contraceptive was important in guiding decision making, and this information was mostly acquired from friends. There was some evidence that the understanding experience was shaped by underlying cultural beliefs.

\section{Influences on family planning decisions}

Influences on decisions about family size

Cultural preference for a male child led some participants to try for further children, encouraged by family members and friends within China, particularly those from more traditional rural communities.

"In China, particularly in rural villages, there is a traditional desire of each family to have a male child. In the UK, I think there is less of this type of cultural view ... previously, my family was in a village, and particularly desired a male child.” [P1]

While this view exerted some influence on decisions made in the UK, the desire to have a larger family was more important for most participants than having further children just to produce a son.

The flexibility to control birth timings was as valued as the freedom to have more children and participants appreciated the autonomy they had in the UK. They contrasted this with China, where they felt contraception or abortions would have been forced upon them. They also discussed having to wait 45 years between pregnancies for a birth permit to be granted. One participant described going into hiding in her second pregnancy and having to pay a large fine. This freedom in the UK was reflected in the participants' childbearing patterns, with closer gaps between children born in the UK than in China and participants all having two or more children.

Perceptions about different contraceptive methods

While in the UK, the most frequently used methods were the IUD, condoms and the implant (Table 1). Our findings highlight the distinctive factors that influence perceptions of each method's advantages and disadvantages. Regarding condoms, the participants noted they were less reliable than other methods but appreciated the flexibility to stop using contraception without first needing to visit a health

Table 1 Contraceptive methods used by the study participants whilst in China and the UK

\begin{tabular}{lllll}
\hline Contraceptive method & $\begin{array}{l}\text { Total (past } \\
\text { and current) }\end{array}$ & Current & In China & In UK \\
\hline Intrauterine device & 8 & 5 & 4 & 6 \\
Implant & 4 & 1 & 0 & 4 \\
Oral contraceptive pill & 1 & 0 & 0 & 1 \\
Condoms & 7 & 3 & 1 & 6 \\
Coitus interruptus & 1 & 0 & 0 & 0 \\
Trying to get pregnant & - & 1 & - & 1 \\
\hline
\end{tabular}

professional and the absence of a direct effect on health or hormone balance.

The IUD was the most commonly used method by those not wanting to have further children. It was a highly trusted long-term option, and the most understood because of its predominant use in China. Participants chose this method because friends had used it and it was perceived as having fewer effects on the body than alternative methods.

"With regards to the coil, because everyone uses it and says that it is very tolerable, so I have also used it." [P8]

Convenience was another key factor but, overall, effects on personal health were the most important consideration. Most participants viewed hormonal methods negatively yet five had used these methods since moving to the UK. Most participants felt they were unnatural and affected the body's balance. Many expressed concerns about effects on their menstrual cycle, a perception often reinforced by friends and family.

\section{"My concern is not that contraception prevents me from having children, but that it is an unnatural method ... because it reduces the volume of menstru- ation from normal which makes it unnatural." [P7]}

Participants said that menstruation should not be affected by contraception. If a method affected its regularity, timing or caused a heavier flow, then it caused an unnatural bodily state and was therefore inappropriate.

\section{DISCUSSION}

\section{Knowledge of contraception}

This study highlighted the distinctive sociocultural factors that compound language barriers and prevent adequate access to family planning care. Studies conducted in China highlight a lack of knowledge about family planning and contraception, ${ }^{19}{ }^{20}$ in particular among the unmarried, migrant youth. ${ }^{33}$ Health professionals need to recognise that younger Chinese women typically come to the UK with very little family planning knowledge and may be at risk of unwanted pregnancy. They may be infrequent health care attenders due to their relative good health and are unlikely to raise the topic themselves. More deliberate strategies may be required to engage these women after arrival in the UK. Women who have already given birth in China may know about and be using the IUD but be unaware of alternatives. There is evidence that extreme long-term use and differences between models used in the UK and China may make removal more difficult. ${ }^{34}$

Our findings suggest that the information provided by the UK health system is inadequate to support our participants' decision-making process. 
Important influences on family planning decision making These women grew up with a state policy that controlled family size and spacing; however, once in the UK this ceases to have an influence. Nevertheless, underlying cultural values continue to shape decision making.

This study supports Wiebe et al.'s ${ }^{10}$ observation that ethnic Chinese women's attitudes towards oral contraceptives are mostly negative. In particular, they feel methods affecting the menstrual cycle are unnatural for the body and therefore undesirable. Our findings draw attention to the specific cultural importance of maintaining natural menstruation and how it strongly influences decision making about contraception. This fits with Chinese cultural beliefs teaching that the natural state of peace and harmony should be sought and that methods of contraception that do not alter the natural rhythm are more acceptable. ${ }^{11}$

\section{Study strengths and limitations}

This is a small study with participants drawn from one city in the UK. It assisted the establishment of trust in the research that the translator who conducted the interviews worked at the clinic where recruitment was initiated and was already known to the women. It did, however, the research team's ability to probe more deeply during interviews. However, our analysis strategy provided an opportunity for interactive discussion to help clarify meaning and so support the trustworthiness of the data collected. ${ }^{27}$

\section{CONCLUSIONS}

This study highlights the need to develop culturally competent strategies to enable access to family planning services for immigrant women from China. It has demonstrated the continuing influence of societal and cultural practices in China on family planning decisions made within the UK. This study provides insight about women from China for those organising family planning services that take the sociocultural background of service users into account. Although this study focused on women from mainland China, for other migrant groups societal and cultural practices are likely to interact with language barriers and unfamiliarity with services, making their experience very different from those educated in the UK, although for each migrant group the way this plays out may be different.

\section{Competing interests None.}

Ethics approval National Research Ethics Committee: West Midlands (Coventry and Warwickshire).

Provenance and peer review Not commissioned; externally peer reviewed.

\section{REFERENCES}

1 Home Office. Immigration Statistics: October to December 2012. https://www.gov.uk/government/publications/ data-tables-immigration-statistics-october-to-december-2012 [accessed 25 December 2013].

2 Hemminki E, Wu Z, Cao G, et al. Illegal births and legal abortions - the case of China. Reprod Health 2005;2:5.

3 Chen YC. Chinese values, health and nursing. J Adv Nurs 2001;36:270-273.

4 Gervais M-C, Jovchelovitch S. The Health Beliefs of the Chinese Community in England: A Qualitative Research Study. London, UK: Health Education Authority, 1998.

5 Johnson MRD, Britain G. Asylum seekers in dispersal: healthcare issues. Home Office Online Report 13/03. 2003. http://library.npia.police.uk/docs/hordsolr/rdsolr1303.pdf [accessed 25 December 2013].

6 Janssens K, Bosmans M, Temmerman M. Sexual and Reproductive Health and Rights of Refugee Women in Europe. Rights, Policies, Status and Needs. Literature Review. Ghent, Belgium: Ghent University, 2005.

7 Keygnaert I, Guieu A, Ooms G, et al. Sexual and reproductive health of migrants: does the EU care? Health Policy, 2014; 114:215-225.

8 Betancourt G. Factors associated with sexual and reproductive health care by Mexican immigrant women in New York City: a mixed method study. J Immigr Minor Health 2013;15: 326-333.

9 Gerrish K, Chau R, Sobowale A, et al. Bridging the language barrier: the use of interpreters in primary care nursing. Health Soc Care Community 2004;12:407-413.

10 Wiebe E, Janssen P, Henderson A, et al. Ethnic Chinese women's perceptions about condoms, withdrawal and rhythm methods of birth control. Contraception 2004;69:493-496.

11 Wiebe ER, Sent L, Fong S, et al. Barriers to use of oral contraceptives in ethnic Chinese women presenting for abortion. Contraception 2002;65:159-163.

12 Dias S, Gama A, Rocha C. Perspectives of African and Brazilian immigrant women on sexual and reproductive health. Eur J Contracept Reprod Health Care 2010;15:255-263.

13 Doherty JP, Norton EC, Veney JE. China's one-child policy: the economic choices and consequences faced by pregnant women. Soc Sci Med 2001;52:745-761.

14 D'Arcangues C. Worldwide use of intrauterine devices for contraception. Contraception 2007;75(6 Suppl.):S2-S7.

15 Chen J, Smith KB, Morrow S, et al. The acceptability of combined oral hormonal contraceptives in Shanghai, People's Republic of China. Contraception 2003;67:281-285.

16 Hoy C. Adolescents in China. Health Place 2001;7:261-271.

17 Tang J, Gao X, Yu Y, et al. Sexual knowledge, attitudes and behaviors among unmarried migrant female workers in China: a comparative analysis. BMC Public Health 2011;11:917.

18 Aresu A. Sex education in modern and contemporary China: interrupted debates across the last century. Int J Educ Dev 2009;29:532-541.

19 Ip WY, Chan MY, Chan DSK, et al. Knowledge of and attitude to contraception among migrant woman workers in mainland China. J Clin Nurs 2011;20:1685-1695.

20 Zhou H, Wang XY, Ye F, et al. Contraceptive knowledge, attitudes and behavior about sexuality among college students in Beijing, China. Chin Med J (Engl) 2012;125: 1153-1157.

21 Chau R, Yu S, Tran C. Understanding the diverse health needs of Chinese people in Britain and developing cultural sensitive services. J Soc Work 2012;12:385-403.

22 Green G, Bradby H, Chan A, et al. 'We are not completely Westernised': dual medical systems and pathways to health care 
among Chinese migrant women in England. Soc Sci Med 2006;62:1498-1509.

23 Bishop FL, Lim CY, Leydon GM, et al. Overseas Chinese students in the UK: patterns and correlates of their use of Western and traditional Chinese medicine. Complement Ther Clin Pract 2009;15:8-13.

24 Green J, Thorogood N. Qualitative Methods for Health Research. London, UK: Sage, 2004.

25 Williamson DL, Choi J, Charchuk M, et al. Interpreterfacilitated cross-language interviews: a research note. Qual Res 2011;11:381-394.

26 Edwards R. A critical examination of the use of interpreters in the qualitative research process. J Ethnic Migration Stud 1998; 24:197-208.

27 Wallin AM, Ahlström G. Cross-cultural interview studies using interpreters: systematic literature review. J Adv Nurs 2006;55: 723-775.

28 Crabtree BF, Miller WF. A template approach to text analysis: developing and using codebooks. Doing qualitative research. Res Methods Prim Care 1992;3:93-109.
29 Orlikowski WJ, Baroudi JJ. Studying information technology in organizations: research approaches and assumptions. Inf Syst Res 1991;2:1-28.

30 Brämberg EB, Dahlberg K. Interpreters in cross-cultural interviews: a three-way coconstruction of data. Qual Health Res 2013;23:241-247.

31 Fereday J, Muir-Cochrane E. Demonstrating rigor using thematic analysis: a hybrid approach of inductive and deductive coding and theme development. Int J Qualitat Met 2006;5:80-92.

32 Delavari M, Farrelly A, Renzaho A, et al. Experiences of migration and the determinants of obesity among recent Iranian immigrants in Victoria, Australia. Ethn Health 2013;18:66-82.

33 Zhao S. Unmet needs for reproductive health knowledge among unmarried migrant youth. J Reprod Contraception 2008;19;227-238.

34 Cheung VY. A 10-year experience in removing Chinese intrauterine devices. Int J Gynaecol Obstet 2010;109:219-222. 